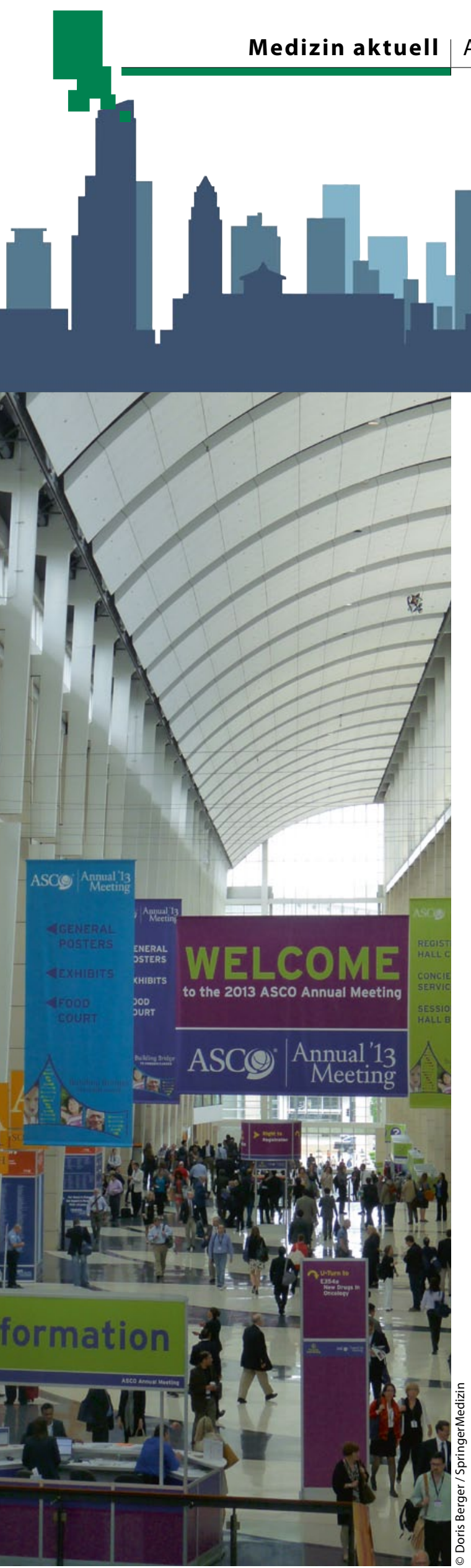

ASCO 2013

\title{
Highlights aus Expertensicht
}

"Building Bridges to Conquer Cancer", so lautete das Motto der diesjährigen Jahrestagung der American Society of Clinical Oncology (ASCO). Vor diesem Hintergrund betonte Monica M. Bertagnolli vom Dana-Farber/Harvard Cancer Center in Boston, MA/USA, die besondere Bedeutung öffentlich geförderter unabhängiger Studien. In den USA werden jährlich immerhin über 200 Millionen US-Dollar öffentliche Gelder für klinische Studien in der Onkologie ausgegeben, die Industrie aber investiert Billionen. Jedoch, so Bertagnolli, mit unterschiedlicher Intention. Die einen, um eine FDA-Zulassung zu erhalten und den Wert des Unternehmens zu steigern, die anderen, um den höchstmöglichen Nutzen für den Patienten und die Gesellschaft zu erreichen. Daher seien öffentlich geförderte Studien so wichtig: um verschiedene Therapiestrategien zu vergleichen, um Therapiemodalitäten zu optimieren, Therapien für seltene Erkrankungen zu entwickeln und nicht zuletzt um Strategien zur Krebsprävention, Früherkennung und zur optimalen Begleitung von Langzeitüberlebenden zu finden. Öffentlich geförderte Studien wurden daher in diesem Jahr besonders präsentiert. Und es gab eine Reihe sogenannter „Investigater-initiated Studies", wie beispielsweise die von Volker Heinemann geleitete FIRE-3-Studie zur Primärtherapie des kolorektalen Karzinoms.

Dennoch - bei über 9.000 Abstracts traten auch diese Beiträge in den Hintergrund. Wie sind die vielen neuen Studienergebnisse aber für die Behandlung der Patienten einzuordnen? Wir haben Experten der jeweiligen Fachgebiete gebeten, die wichtigsten Daten vor dem Hintergrund der Versorgungsrealität in

Deutschland darzulegen. 